\title{
La mesure des débits d'eau dans les conduites fermées par la méthode de temps de transit d'un traceur radioactif
}

PAR

\author{
L. Roche \\ Chef de Département adjoint \\ à la Direction des Etudes et Recherches d'Electricité de France
}

La recherche de moyens opérationnels de mesurage ou de contrôle de débit, en conduites fermées, de mise en oeuvre simple et au moindre coût, a conduit la Direction des Etudes et Recherches d'Electricité de France, a développer les méthodes de mesure par traceurs radioactifs.

Ces dernières présentent les avantages suivants :

- Elles n'occasionnent aucune perturbation de l'écoulement du fluide,

- Elles n'entraînent aucune perte de charge supplémentaire,

- Elles ne nécessitent en général aucune modification des circuits,

- Elles n'exigent pas d'immobilisation temporaire de l'installation.

Elles ont conduit à l'élaboration et la mise au point de matériels originaux couramment utilisés en essais lorsque les moyens conventionnels ne sont pas adaptés.

La méthode retenue est la mćthode du tcmps de transit appelée également "méthode d'Allen" et dont la théorie fera l'objet d'un exposé séparé. Nous en rappellerons le principe.

\section{Principe de la méthode}

La mesure du débit par la méthode du temps de transit est fondée sur la mesure du temps de transit de particules "marquées" entre deux sections droites de conduite éloignées d'une distance connue. Le marquage des particules est réalisé par injection d'un traceur dans l'écoulement, en amont des deux sections de mesure. Le temps de transit est obtenu en faisant la différence des temps moyens d'arrivée du traceur au niveau de chacune des positions de détection.
Le débit $q$ est donné par la relation :

$$
q=\frac{V}{\bar{t}}
$$

où $\bar{t}$ est le temps de transit de particules marquées,

$V$ est le volume de la conduite entre les deux sections de détection

En pratique, on obtient la valeur de $\bar{t}$ en mesurant la différence des centres de gravité temporels des nuages de particules marquées lors de leur passage devant les détecteurs.

Le signal issu de ces derniers doit être proportionnel à l'activité volumique du traceur mais il n'est toutefois pas nécessaire de connaître la valeur du coefficient de proportionnalité et par conséquent, la valeur absolue de la concentration.

Cette méthode est actuellement en cours d'étude dans le cadre de l'organisation internationale de normalisation (I.S.O.). C'est le sous-comité ISO/TC 30/SC 4 qui en est chargé.

\section{Choix du traceur radioactif}

Le traceur doit satisfaire à deux impératifs :

- Le premier d'être émetteur d'un rayonnement suffisamment énergétique et intense pour traverser les parois et exciter le détecteur placé à l'extérieur de la conduite.

- Le second, de ne présenter aucun risque radioactif.

L'énergie du rayonnement émis doit être de l'ordre de plusieurs centaines de kilo électron-volt (keV) et l'activité volumique du fluide, après dilution, largement supérieure au seuil minimal de détection des sondes industrielles. L'ac- 
tivité du traceur diminue au cours du temps et, ceci d'autant plus vite que sa période est plus courte.

Or, les délais de transport, la préparation et la mise en ceuvre du traceur sur les lieux d'intervention nécessitent d'approvisionner des radioéléments de période de plusieurs heures, voire de plusieurs jours pour disposer d'une activité suffisante au moment des mesures.

La législation a défini, pour chaque radioélément, une activité maximale à ne pas dépasser qui est appelée "concentration maximale admissible" et au-dessous de laquelle le fluide marqué ne présente aucun risque pour l'homme.

On peut observer que d'une manière générale la nuisance d'un radioélément augmente avec l'activité et avec la période.

Pour résoudre un tel compromis, en tenant compte de ces deux impératifs contradictoires, les générateurs de radioéléments apportent une solution élégante.

Faisant appel aux propriétés radioactives des "couples de filiation", ils permettent d'obtenir, à partir d'un radioélément à période longue, un radioélément à vie courte.

Nous avons mis au point des matériels mettant en œuvre ce principe, utilisant différents "couples", délivrant "in situ" le traceur radioactif le mieux adapté et concourant ainsi directement à rendre opérationnelles ces méthodes de mesurage de débit de fluide liquide.

Le générateur-injecteur actuellement opérationnel à Electricité de France utilise le "couple" césium-baryum. (figure 1)

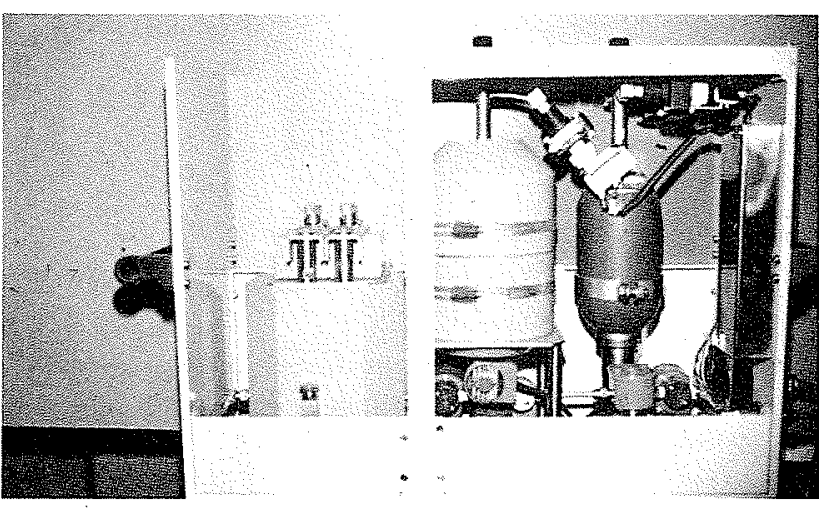

$1 /$ Générateur

\section{Principe}

L'élément père, le césium 137 de période 30 années se désintègre en émettant un rayonnement Béta et en donnant naissance à son fils, le baryum 137, métastable et émetteur de photons $\gamma$ d'énergie de $662 \mathrm{keV}$ qui seront détectés.

Le baryum $137 \mathrm{~m}$ retrouve ainsi, par désexcitation, un état stable avec une période de 2,6 minutes.

\section{Obtention du traceur}

Le césium 137 est fixé sur des résines anioniques ; la séparation chimique et l'extraction du baryum $137 \mathrm{~m}$ sont obtenues par élution de ces résines avec une solution de nitrate de calcium. Cette solution entraîne le baryum et constitue le "traceur radioactif" qui sera injecté dans la conduite.
Le processus chimique d'extraction du baryum et son injection sous forme d'une solution aqueuse de nitrate de calcium + nitrate de baryum se font automatiquement à partir du générateur-injecteur commandé à distance.

\section{Technologie}

L'appareil a été développé avec un souci de simplicité, de frabilité, de moindre coūt et de mise en station facile.

Le radioélément père est contenu dans une cartouche appelée "cartouche active". Cette dernière, composée d'un fourreau cylindriquue en acier inoxydable, enferme les résines anioniques de granulométrie connue, chargées en ferrocyanure double de $\mathrm{K}$ et $\mathrm{Cu}$, sur lesquelles est fixé le césium 137.

Ces résines sont maintenues compactes en milieu constamment aqueux entre deux "bouchons filtres", en métal fritté, solidaires mécaniquement du fourreau et permettant le passage d'un débit continu et optimal de solution éluante.

Une deuxième cartouche, réalisée suivant le même principe mais sans césium fixé, appelée "cartouche de garde" est placée sur le circuit d'injection. Elle fixe les éventuelles traces d'élément père qui pourraient être entrainées lors de l'élution et empêche ainsi la contamination en césium du traceur.

Une pompe volumétrique assure la circulation du nitrate de calcium en solution à travers les cartouches et achemine la solution aqueuse de traceur résultante vers un accumulateur hydropneumatique qui assure l'injection instantanée du traceur dans la conduite en charge.

Un ensemble d'électromécanisme permet le déroulement automatique des différentes phases.

L'unique pompe du système sert à l'élution des cartouches et au stockage momentané de "la solution traceur" dans l'accumulateur hydropneumatique dont la pression de gonflage est règlable, en fonction de celle du fluide, dans la conduite au point d'injection.

Les deux cartouches sont placées à l'intérieur de l'ensemble générateur-injecteur dans un conteneur en plomb étudié spécialement. Il assure la protection radiologique et un remplacement aisé des cartouches suivant certaines précautions.

Les analyses du fluide, faites périodiquement à partir de prélèvements opérés à la sortie de l'usine, pendant les mesures, confirment l'absence totale de contamination des eaux de rejet.

La mise en place de l'ensemble des moyens sur le site est rapide et les conditions de mise en oeuvre sont d'une grande simplicité.

\section{Sécurité - Radioprotection}

Le matériel présente les garanties indispensables à la protection du personnel contre :

- L'irradiation externe en période d'arrêt, d'entretien et de fonctionnement de l'installation,

- l'irradiation interne ou externe résultat de la contamination de l'eau au domaine public.

En tout premier lieu, l'activité de la cartouche reste, dans le cas général, très faible (de l'ordre d'une centaine de millicuries) ; elle est placée dans un conteneur qui assure la pro- 
tection radiologique et le débit d'exposition à la surface de l'appareil est bien inférieur à la valeur légalement permise.

$\mathrm{Au}$ voisinage immédiat de l'appareil, il est de l'ordre de celui que l'on peut relever dans certaines régions, telles que l'Auvergne ou la Bretagne et qui est consécutif à la radioactivité naturelle.

Au point d'injection, l'expérience montre que sur le plan de la contamination, deux contaminants sont à considérer :

- Le $137 \mathrm{Ba}^{\mathrm{m}}$ qui est le traceur utilisé,

- Le 137 Cs qui est retenu dans la cartouche par fixation sur des résines.

La concentration maximale admissible pour le public (CMAP) du 137 Ba est au moins égale à $5000 \mu \mathrm{Ci} / \mathrm{m}^{3}$; l'activité volumique maximale rejetée a été trouvée au rejet, lors d'un essai en usine, égale à $250 \mu \mathrm{Ci} / \mathrm{m}^{3}$ pour une activité injectée de $200 \mathrm{mCi}$ et pour un débit de $2 \mathrm{~m}^{3} / \mathrm{s}$.

Cette valeur est 20 fois plus faible que la C.M.A.P. laquelle est calculée pour une exposition continue ( 24 heures sur 24) des personnes alors qu'il s'agit, ici, d'une exposition limitée à quelques minutes.

En ce qui concerne le $137 \mathrm{Cs}$, la fuite éventuelle a été réduite en plaçant, en aval de la cartouche active, une cartouche de garde, de même nature que la précédente. Des contrôles périodiques sont effectués à la sortie du générateur et après dilution. Les contrôles effectués jusqu'à ce jour, en particulier après un millier d'injections montre que la contamination était inférieure à $10^{-8} \mathrm{Ci}$ pour une solution de traceur de $50 \mathrm{~cm}^{3}$.

Ces $10^{-8} \mathrm{Ci}$ sont dilués, à la sortie de l'usine, dans un volume de l'ordre de $100 \mathrm{~m}^{3}$, ce qui correspond à une largeur de nuage de 50 secondes et un débit de $2 \mathrm{~m}^{3} / \mathrm{s}$.

L'activité volumique résultante de $10^{-10} \mathrm{Ci} / \mathrm{m}^{3}$ est $10^{-5}$ fois inférieure à la concentration maximale admissible du 137 Cs dans l'eau de boisson, pour les personnes du public.

Ce résultat a pu être obtenu grâce à une élaboration très poussée de la cartouche et de ses filtres.

Pour accroître encore les marges de sécurité, il a été prévu la possibilité d'un changement aisé et peu onéreux de cartouche de protection.

\section{Mesurage du temps de transit}

Les rayonnements sont détectés au fur et à mesure de leur apparition et les signaux sont traités pour obtenir le centre de gravité et en déduire le temps de transit.
Cette solution présente de gros avantages par rapport à l'exploitation directe des enregistrements analogiques, longue, fastidieuse et peu précise.

\section{Unité de détection}

Elle est constituée d'une chaine à scintillation classique qui comporte :

- Un scintillateur,

- Un photomultiplicateur.

\section{Unité de traitement (figure 2).}

Ce dispositif fonctionnant en temps réel permet la mesure du temps qui sépare l'injection du passage du nuage radioactif au droit d'une section de mesure ou entre deux sections de mesure.

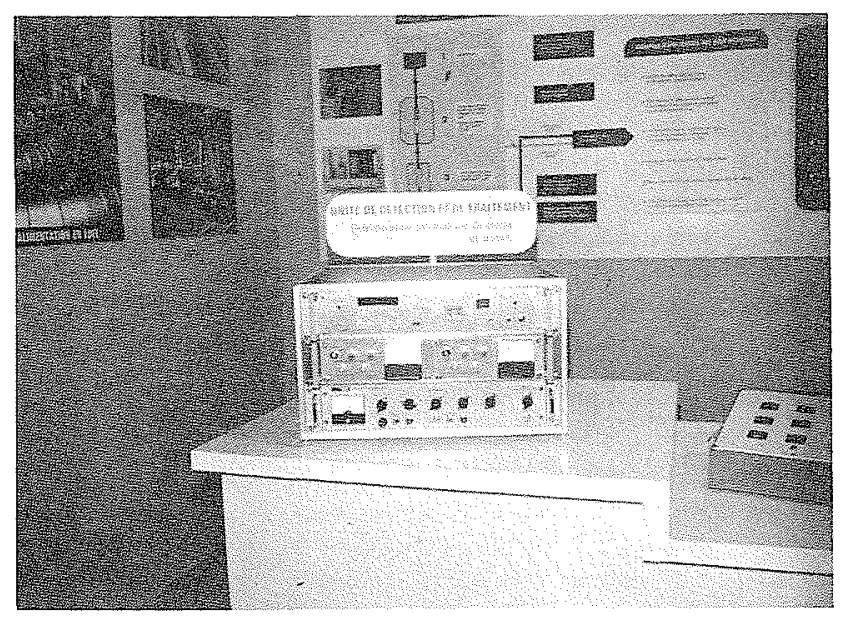

2/ Unité Détection

L'unité de traitement élabore le centre de gravité temporel du rayonnement reçu entre les détections de début et de fin du nuage. Elle calcule ensuite le temps qui sépare ces deux centres de gravité (le premier centre de gravité pouvant être remplacé par le signal d'injection).

\section{Discussion}

Président : M. Roger Roche

M. Le Président remercie M. L. RochE de son intéressant exposé et ouvre le débat.

M. DOUillet (D.T.G., E.D.F., Grenoble) intervient en ces termes :

"Pour compléter l'exposé de M. L. Roche, Je pense qu'il est utile de donner quelques indications sur les utilisations industrielles de la méthode des temps de transit et de l'appareil Générateur Injecteur de Radio-Eléments pour des mesures de débit.
"La commodité d'utilisation de ce type d'appareillage par des équipes non spécialisées dans l'emploi de radio-élément a permis de réaliser de nombreuses mesures ponctuelles dans des installations industrielles de toute importance. Les responsables de la détermination des caractéristiques hydrauliques des installations de production d'énergie par exemple disposent ainsi d'un nouveau moyen, qui vient compléter la panoplie de ceux dont ils disposaient depuis longtemps : moulinets, pitots, méthode thermodynamique de mesures des rendements, etc. et qui, dans certains 
cas, est d'un emploi plus précis et plus pratique. Dans quelques cas, des mesures comparatives avec deux méthodes utilisées conjointements ont pu être réalisées, qui ont défini la validité de la méthode.

"Une autre utilisation a été expérimentée dans une centrale hydro. électrique en vue de contrôler, de manière très fine, l'évolution du rendement d'une turbine de centrale hydro-électrique en fonction du temps sur une longue période : l'appareillage, monté à poste fixe sur la conduite forcée, permettait une mesure depuis le tableau de l'usine, du débit dans des conditions d'ouverture géométrique de l'organe de réglage du débit et de chute définies avec une excellente précision. La dispersion des mesures réalisées sur une période de plusieurs mois est restée inférieure à $0,2 \%$ parfaitement compatible avec la stabilité parfaite de l'état mécanique de la turbine pendant cette période, ce qui démontrait l'excellente fidélité de cette méthode".

Lorsque le diamètre de la conduite dépasse 40 à 50 centimètres, ajoute M. L. RochE, l'emploi d'un détecteur, placé à l'extérieur de celleci peut rendre les mesures difficiles. En effet, pour le "caesium-barium" par exemple, au-delà d'une distance de 60 centimètres, les particules radioactives ne sont plus prises en compte par le scintillateur. Dans de tels cas, la solution idéale est de disposer d'un "puits" permettant de placer le détecteur au centre de l'écoulement.

Pourtant remarque $M$. GUIZERIX, je me souviens d'avoir fait avec votre prédécesseur $M$. WOLF, des mesures parfaitement valables dans une conduite de 1 mètre de diamètre ; mais il faut pour cela disposer d'une longueur de conduite suffisante entre le point d'injection et la première section de mesure.

Répondant à une question de M. CA ZENAVE, M. DOUILLET précise que ses expériences de contrôle permanent des débits (et des rendements) ont été faites sur une turbine Pelton, alimentée par une longue conduite lorsque l'on dispose d'une longueur de conduite égale à environ cent fois le diamètre de celle-ci -ce qui est très fréquent dans les usines de moyenne et de haute chute- la condition de bon mélange (essentielle pour le succès de la méthode) est largement respectée.

M. CRAvERo fait par des résultats de mesures de débits effectués par M. L. Roche à la Centrale thermique de Cordemais concurrement par la méthode ALLEN avec des traceurs radioactifs et par celle des moulinets. Malgré les difficultés dues au fonctionnement de ces derniers dans l'eau de mer, l'écart entre les débits mesurés respectivement par ces deux méthodes est resté dans la fourchette $1.0,5 \%$ (moyenne pondérée). J'ai été surpris, dit M. CRAVERO, de ces résultats car la première section de mesure se trouvait à 60 mètres seulement du point d'injection et le diamètre de la conduite dépassait 2 mètres. Si l'on a obtenu d'assez bons résultats, cela est peut être dû au dispositif de mesure qui comportait, je crois, 4 détecteurs disposés suivant un diamètre de la conduite
A Cordemais, précise M. L. ROCHE, l'injection se faisait à l'aspiration de la pompe de circulation de l'eau des condenseurs. Après des essais peu satisfaisants effectués à Fessenheim au moyen de détecteurs placés à l'extérieur de la conduite, il s'est avéré que la précision des mesures était nettement améliorée en immergeant les détecteurs dans la conduite comme il a été dit plus haut. A l'occasion de mesures réali sées sur des tuyauteries cylindriques Bonna, des puits ont été aménagés à l'intérieur de la conduite; chacun d'eux a été équipé de 4 détecteurs dont les réponses ont été soigneusement analysées. C'est à partir des enseignements tirés de ces essais …encore en cours de développement qu'ont pu être réalisées les mesures de Cordemais, évoquées par M. CRAVERO.

Sur une remarque de M. MARgrita, M. Le Président observe "L'erreur sur la mesure du temps n'a pas plus d'importance que celle sur la mesure du volume. Il faut donc connaitre le volume avec précision. S'il s'agit d'une installation neuve, c'est relativement facile ; je dis bien "relativement", car les dimensions des tuyauteries ou celles des galeries importantes ne sont pas tellement faciles à établir. Il y a en effet un problème de mesure du volume.

J'avais cru comprendre, dit M. MOLINARI, que l'un des objectifs de l'E.D.F., dans le cas de certains essais relatés par M. DoulLLET, n'était pas de faire des mesures absolues de débit, mais d'observer l'évolution de rendements de turbines en procédant à des mesures systématiques et répétitives dans les mèmes conditions. Dans ce cas, la justesse de la mesure importe peu, ce qui compte, c'est la fidélité du dispositif.

Effectivement, confirme M. L. ROCHE, la fidélité du système de mesure peut être suffisante pour certaines applications; mais dans d'autres cas, il est nécessaire de mesurer avec précision la valeur absolue des débits. Il en est ainsi notamment lors des essais de réception ou de mise au point des turbines et des pompes.

L'un des avantages de la méthode que nous avons décrite est de ne pas exiger un arrêt toujours relativement long des machines à essayer ainsi que cela est nécessaire pour l'installation et le démontage des moulinets dans la section de mesure lorsque le débit est déterminé par exploration du champ des vitesses.

Lorsqu'il s'agit de mesurer des débits d'eau très chaude à des températures voisines de la saturation l'emploi des traceurs est particulièrement indiqué, car il n'est pas possible d'utiliser dans ce cas les appareils déprimogènes (diaphragmes, tuyères, Venturis).

En l'absence d'autre intervention, la discussion est close et $\mathrm{M}$. Le Président donne la parole à M. Le Professeur P.-Ch. LEvEQue de la Faculté des Sciences de Bordeaux pour l'exposé de sa Communication.

\section{Abstract \\ Determination of pipe discharge by radioactive tracer transit time measurement}

Electricité de France has developed a number of simple, inexpensive radioactive tracer methods for pipe discharge measurement. The equipment is highly adaptable and causes neither flow disturbance nor additional loss of head, nor does it require modification of existing circuits as a general rule.

The method described is based on the fluid transit time between two detection stations along the pipe. Discharge is found by dividing the volume between the two measurement stations by the time the fluid takes to transit from one to the other. To determine transit time, the fluid is "marked" by a tracer with a short half-life, which is produced and fed into the pipe flow by a special "generator/injector" unit.
Caesium 137, a radio-element with a long half-life (30 years), produces metastable barium 137, which emits gamma-radiation at 662 $\mathrm{keV}$ with a half-life of 2.6 minutes. The caesium is fixed on resin inside a cartridge sealed with filter plugs at bothends. A positive-displacement pump circulates the fluid extracting the barium 137 and stores the resulting aqueous tracer solution in a hydropneumatic accumulator.

Direct transit time indication is provided by an electronic detection and processing unit.

This equipment is designed to current French regulations, and the "marked" fluid is safe as regards radioactivity. 